\title{
Kibble-Zurek Scaling during Defect Formation in a Nematic Liquid Crystal
}

DOI:

10.1002/cphc.201700023

\section{Document Version}

Accepted author manuscript

Link to publication record in Manchester Research Explorer

\section{Citation for published version (APA):}

Fowler, N., \& Dierking, I. (2017). Kibble-Zurek Scaling during Defect Formation in a Nematic Liquid Crystal. ChemPhysChem. https://doi.org/10.1002/cphc.201700023

\section{Published in:}

ChemPhysChem

\section{Citing this paper}

Please note that where the full-text provided on Manchester Research Explorer is the Author Accepted Manuscript or Proof version this may differ from the final Published version. If citing, it is advised that you check and use the publisher's definitive version.

\section{General rights}

Copyright and moral rights for the publications made accessible in the Research Explorer are retained by the authors and/or other copyright owners and it is a condition of accessing publications that users recognise and abide by the legal requirements associated with these rights.

\section{Takedown policy}

If you believe that this document breaches copyright please refer to the University of Manchester's Takedown Procedures [http://man.ac.uk/04Y6Bo] or contact uml.scholarlycommunications@manchester.ac.uk providing relevant details, so we can investigate your claim.

\section{OPEN ACCESS}




\title{
Kibble-Zurek scaling during defect formation in a nematic liquid crystal
}

\author{
N. Fowler, I. Dierking* \\ School of Physics and Astronomy, University of Manchester, Oxford Road, \\ Manchester M13 9PL, United Kingdom
}

\section{Abstract}

Symmetry breaking phase transitions are often accompanied by the formation of topological defects, like in cosmological theories of the early universe, superfluids, liquid crystals or solid state systems. This scenario is described by the Kibble-Zurek mechanism, which predicts respective scaling laws for the defect density $\rho$. One such scaling law suggests a relation $\rho \sim \tau_{\mathrm{Q}}^{-1 / 2}$ with $\tau_{\mathrm{Q}}$ the change of rate of a control parameter. In contrast to the scaling of the defect density during annihilation with $\rho \sim \mathrm{t}^{-1}$, which is governed by the attraction of defects of the same strength but opposite sign, the defect formation process, which depends on the rate of change of a physical quantity initiating the transition, is only very scarcely investigated. We here use nematic liquid crystals as a different system to demonstrate the validity of the predicted scaling relation for defect formation. It is found that the scaling exponent is independent of temperature and material employed, thus universal, as predicted.

Topological defects[1] are often generated following symmetry breaking phase transitions in many aspects of physics including cosmology, superfluids and liquid crystals. Kibble proposed a universal mechanism by which cosmological defects were formed in the early universe as it expanded and cooled shortly after the Big Bang[2.3]. During a cosmological symmetry breaking phase transition, uncorrelated domains with randomly determined order are produced which subsequently coalesce. Defects are formed that exist between two domains with non-compatible order. The initial density of defects is dependent on the rate at which domains grow which is limited by the finite speed of information transmission (causality). It became known as the "Kibble-Zurek mechanism" after Zurek suggested that 
the scenario should also be applicable for condensed matter systems[4]. Zurek derived a number of universal scaling laws which describe defect dynamics and proposed an experiment involving vortex formation in superfluid helium to provide confirmation ${ }^{4}$. Two such scaling laws of interest in relation to this paper describe the formation and annihilation of defects, given by equation (1)[4] and (2)[5], respectively:

$$
\begin{aligned}
& \rho \propto \tau_{Q}{ }^{-\frac{1}{2}} \\
& \rho \propto t^{-1}
\end{aligned}
$$

where $\rho$ is the defect density, $\tau_{Q}$ is the electric field ramp rate and the time after the transition.

Computational simulations as well as numerical studies have confirmed the exponents given in both scaling laws[6,7]. The latter scaling exponent relating to defect annihilation has been confirmed experimentally - also using liquid crystals[8,9]. The scaling law of defect formation, eq.(1), has been much less investigated experimentally, although the KibbleZurek mechanism has been investigated in numerous condensed matter systems including liquid crystals8,9, superfluid ${ }^{3} \mathrm{He}[10,11]$ and ${ }^{4} \mathrm{He}[12,13]$ as well as in solid state systems such as Josephson junctions[14] and ionic crystals[15]. Confirmation of eq.(1) was found by Ducci et al. for a nonlinear optical system based on the Kerr effect[16], by Monaco et al. for spontaneous vortex formation in Josephson tunnel junctions[17], and by Chae et al. in a solid state ferroelectric system[18]. Also in recent years, the dynamic behaviour of defects has attracted attention, although mainly the annihilation process was investigated. Bogi et al.[19] observed two annihilation regimes for $\pm 1 / 2$ defect pairs, a linear defect velocity regime, where the elastic interaction between defects is screened by the anchoring energy and a late time regime, close to annihilation where a 1/2-power law was observed for the distance versus time plots. Oswald et al.[20] demonstrated that backflow was the cause of an observed anisotropy of defect motion in the annihilation process. Both observations were also confirmed by Dierking et al.[21] for $s= \pm 1$ umbilic defects. Very recently, defect annihilation studies were extended to smectic C thin film systems, where it was shown theoretically[22] and experimentally[23] that the process is strongly dependent on the actual values of the splay and bend elastic constants. Experimental and theoretical results so 
far have recently been summarized in a review by del Campo and Zurek[24]. For liquid crystals the process of defect annihilation (eq.(2)) has thus extensively been studied experimentally, however, the process of defect formation (eq.(1)) was not.

The simplest of the liquid crystals[25] is the nematic phase, which solely exhibits orientational order described by the director field $n(r)$, while the molecular centres of mass are isotropically distributed. String defects generated in a nematic liquid crystal following pressure quenches were shown to annihilate inversely with time[8], $\rho^{\sim} t^{-1}$. Further confirmation for the defect annihilation scaling was achieved using electric field induced defects generated in a nematic liquid crystal.[9] These so called umbilical defects[26-28] do not end in a singular point, but are otherwise very similar to the Schlieren defects. They only differ in so far as the director continuously tilts into the direction of the substrate normal, being parallel to it at the defect core, which is small, but of finite size. Also in the present work, defects were generated via a Fréedericksz transition in a nematic liquid crystal with negative dielectric anisotropy $(\Delta \varepsilon<0)$ confined by homeotropic boundary conditions (director perpendicular to the bounding glass substrates). Application of an electric field will in this case induce a switching transition to planar orientation, accompanied by the formation of defects. Such defects are localised regions of escape whereby the director field is reoriented parallel to an electric field. The in-plane component of the director rotates through $2 \pi$ resulting in a topological defect strength of $s= \pm 1$ (fig. 1).

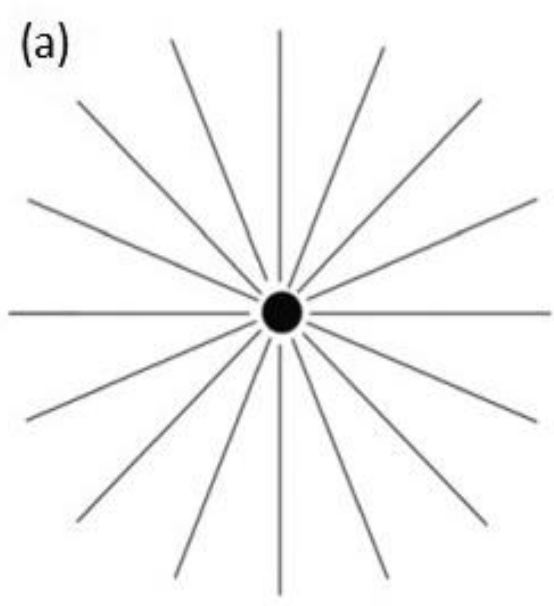

$\mathrm{s}=+1$

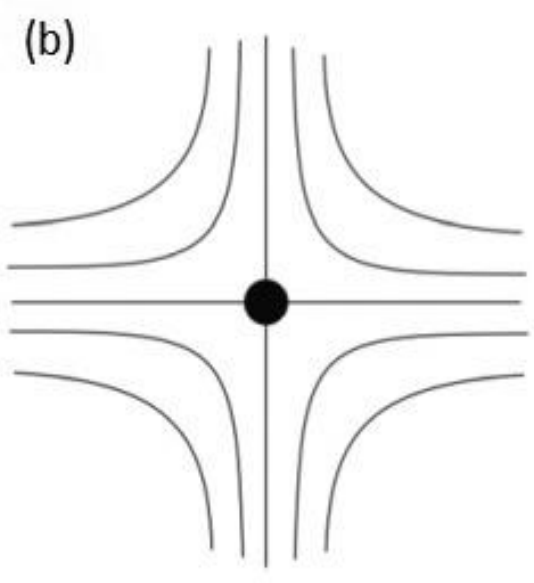

$S=-1$ 
Figure 1. Schematic director field around $a(a) s=+1$ and (b) $s=-1$ topological defect. The defect core is indicated by the black dot in the middle. In the experiment, both types of defects are formed at an equal amount, contributing to the defect density.

It should be pointed out that the Fréedericksz transition is not a thermodynamic phase transition such as the isotropic to nematic transition, which is generally weakly first order, and often used in quench experiments. It is a continuous texture transition at constant temperature within the nematic phase, induced by the application of an electric (or magnetic) field. While for the induction of defects for crossing a thermodynamic phase transition, the temperature needs to be changed at different cooling rates $d T / d t$, we here change the rate of electric field application, $\mathrm{dE} / \mathrm{dt}$, i.e. the ramp rate, when inducing defects at constant temperature.

The liquid crystal used in this investigation was a nematic mixture ZLI-2806, supplied by Merck, Darmstadt. ZLI-2806 has a negative dielectric anisotropy of $\Delta \varepsilon=-4.8$ and its phase sequence on cooling is: Isotropic $100{ }^{\circ} \mathrm{C}$ Nematic $-20^{\circ} \mathrm{C}$ Crystal. Standard sandwich cells with thickness $10 \pm 0.1 \mu \mathrm{m}$ and homeotropic boundary conditions (promoted by cetyl trimethylammonium bromide, CTAB) were used, according to the sandwich cell construction schematically shown in figure 2 . These act like a plate capacitor with the electric field applied perpendicular to the substrate planes by thin wires.

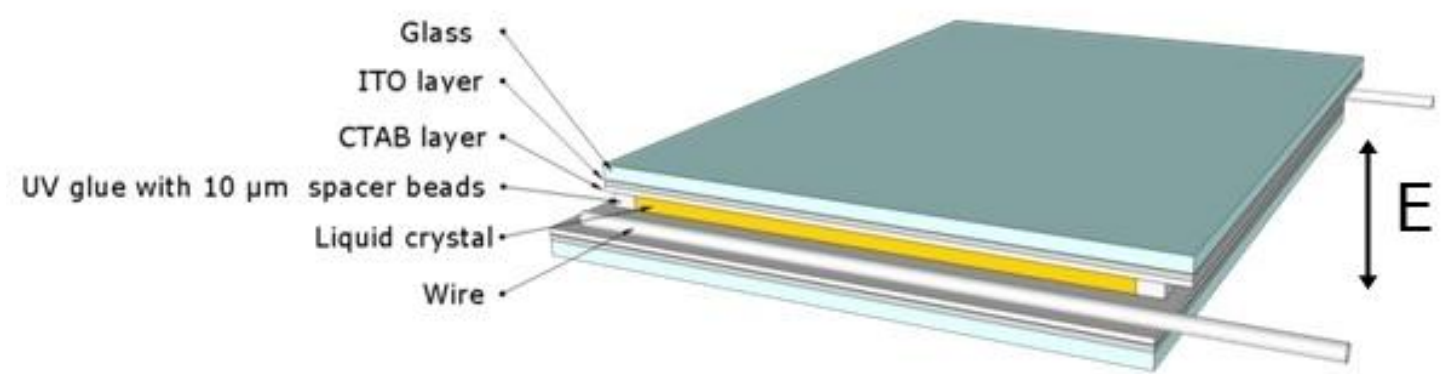

Figure 2. Sandwich cells were built using two ITO coated glass plates upon which CTAB was adsorbed. The cells were held together with UV glue containing $10 \mu \mathrm{m}$ spacer beads and 
sealed using an epoxy resin. Thin wires, soldered to the electrodes, were used to apply electric fields.

Defects were generated with an electric field above the threshold field of $E_{t h}=0.2 \mathrm{MV} / \mathrm{m}$, applied across the cell. The electric field was linearly ramped to a maximum amplitude of 0.8

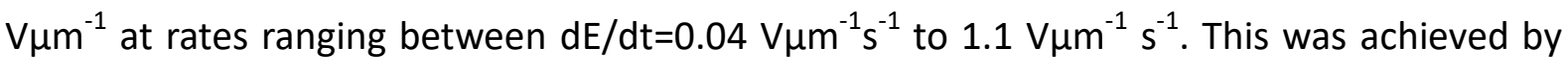
using a TTi TGA12101 waveform generator to modulate the amplitude of a sinusoidal carrier wave with frequency $f=1 \mathrm{kHz}$, produced using a HP 3312A waveform generator. The high frequency sinusoidal carrier wave was used to prevent electro-convection which could affect defect formation as well as ion transportation which could screen the electric field applied across the cell. The temperature was held constant by a Linkham TMS-91 hot stage and controller which has a relative precision of $0.1 \mathrm{~K}$ at an absolute temperature accuracy of $1 \mathrm{~K}$. Defect formation was viewed in a polarising microscope (Nikon Optiphot-pol) and captured using a digital camera (IDS uEye GigE). The digital camera recorded frames at a resolution of $640 \times 480$, corresponding to an image size of $0.243 \mathrm{~mm}^{2}$, at a frame rate of 100 frames per second. We note that the Fréedericksz transition is a dielectric process, where the torque on the director is proportional to $\Delta \varepsilon \mathrm{E}^{2}$. In other experiments of this type the formation of defects is introduced by linearly changing an intrinsic variable of state across a transition from a uniform to a non-uniform (defect) state. Often temperature is used, such that $d T / d t$ provides the change in external variable. Accordingly, we have used the field ramp $\mathrm{dE} / \mathrm{dt}$ as the variable introducing the defect formation, where $\tau_{Q}$ of eq.(1) is the electric field ramp rate. A universal mechanism of defect formation should be independent of the physical system studied, and the variable that is changed to induce the formation of defects.

Defects, which are visible in the polarising microscopic textures as the cross points between four dark brushes as indicated in fig. 3 , were counted manually at a time of $t=0.25$ seconds from the onset of the Fréedericksz transition with the aid of imaging software IMAGETOOL 3.0, developed at the University of Texas Health Science Center. The software does not rely on an automated algorithm to count defects, but simply indicates and successively counts the defects that are marked. The defect density is then calculated from the number of defects per unit area as an average of 15 repeat measurements. It should be noted that 
generally, defects are randomly formed at arbitrary positions in the field of view, with only very few defects forming at the same spatial position in successive experiments. Influences of cell substrate non-uniformities on the defect patterns were further minimized by averaging over experimental runs carried out with different cells of equal cell gap under otherwise equal conditions. The time delay before measurement of the defect density was required in order for the defects to become sufficiently defined so that they could clearly be identified. At the same time, it needed to be assured that the subsequent defect annihilation had not taken place in any significant manner. $t=0.25 \mathrm{~s}$ after the Fréedericksz transition was the optimum time to assure both completed defect formation and mostly frozen defect dynamics for annihilation. Figure 3 depicts the development of defects

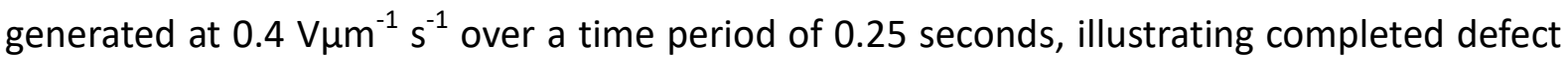
formation, with only negligible defect annihilation present. An equivalent behaviour was observed for other electric field ramp rates.

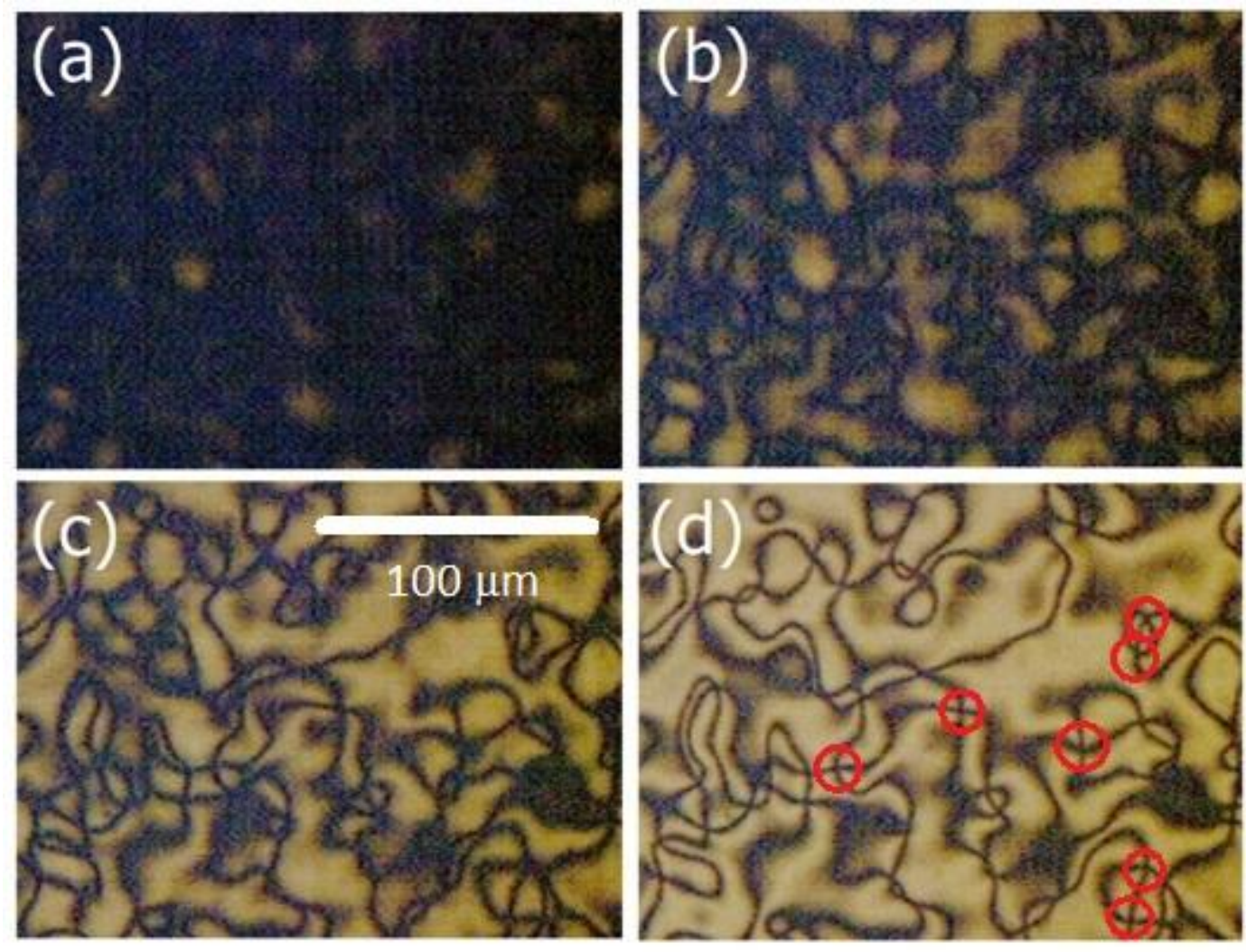


Figure 3. Defect formation during the electric field induced director reorientation at (a) $t=0.05 \mathrm{~s}$, (b) $t=0.1 \mathrm{~s}$, (c) $t=0.15 \mathrm{~s}$ and (d) $t=0.25 \mathrm{~s}$, at which time the defect density was determined. Some of the defects are exemplary indicated in part (d) by red circles.

Figure 4 depicts the density of defects formed in liquid crystal ZLI-2806 during ramps

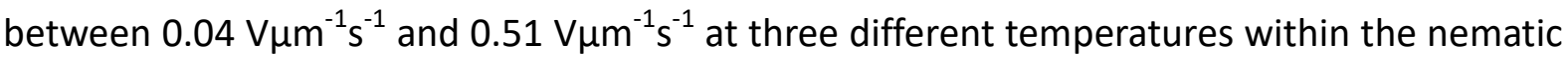
phase. Reduced temperatures are stated with respect to the nematic-isotropic transition temperature, $\mathrm{T}_{\mathrm{N} I}$. Each data point constitutes the average of 15 repeat measurements. The standard error was found to be smaller than the size of the symbols. The error bars stated represent the error associated with counting the number of defects. The scaling exponent was measured by determining the slope of the line. The dashed line represents the predicted scaling exponent $\alpha=-1 / 2$ from eq.(1). Measured scaling exponents are independent of temperature and found to be in excellent agreement with the predicted value, as depicted in the inset of figure 4.

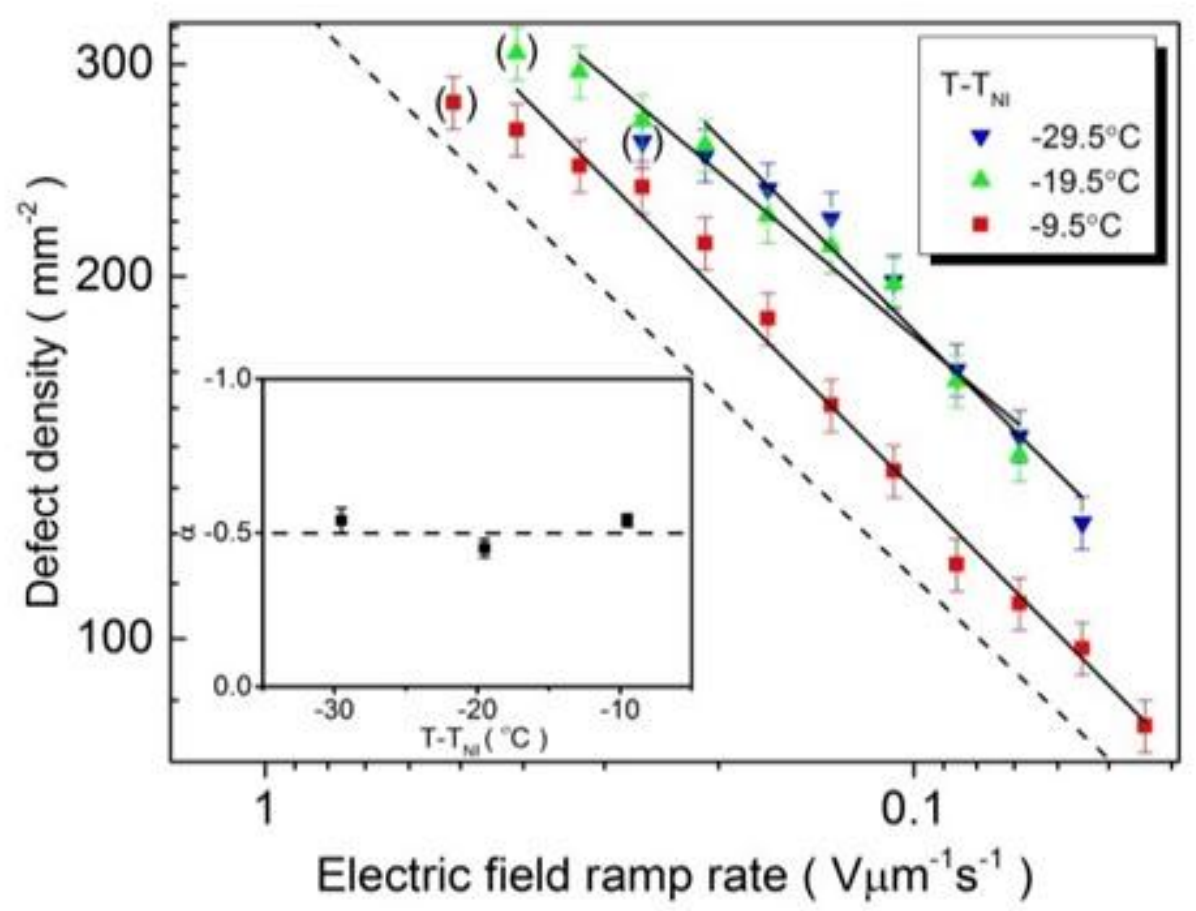

Figure 4. Defect density as a function of electric field ramp rate for the defect formation in ZLI-2806 at three different temperatures. The inset demonstrates the indepence of the scaling exponent $\alpha$ on reduced temperature $T-T_{N I}$ within the nematic phase. 
The data points in brackets indicate regimes where the scaling deviated from that expected. The density of defects generated at lower electric field ramp rates was sufficiently low that defects did not have time to move towards each other and annihilate before their density was measured. However, at larger defect densities, generated at higher ramp rates, defects had begun to annihilate before their density could explicitly be measured. This implies that at these higher defect densities their annihilation dynamics is not frozen out, so that data in this regime needs to be ignored for the determination of the scaling exponent $\alpha$ according to equation (1). It is clear that this ramp rate regime changes with temperature, due to a change of liquid crystal viscosity.

The experiment was also repeated with a shorter time delay of 0.1 seconds instead of $0.25 \mathrm{~s}$ before measurement of the defect density. The results depicted in figure 5 show the exponent of scaling as expected before, deviating once more and confirming that defect annihilation is the reason for the deviation from the predicted scaling behaviour at large ramp rates, i.e. large defect densities.

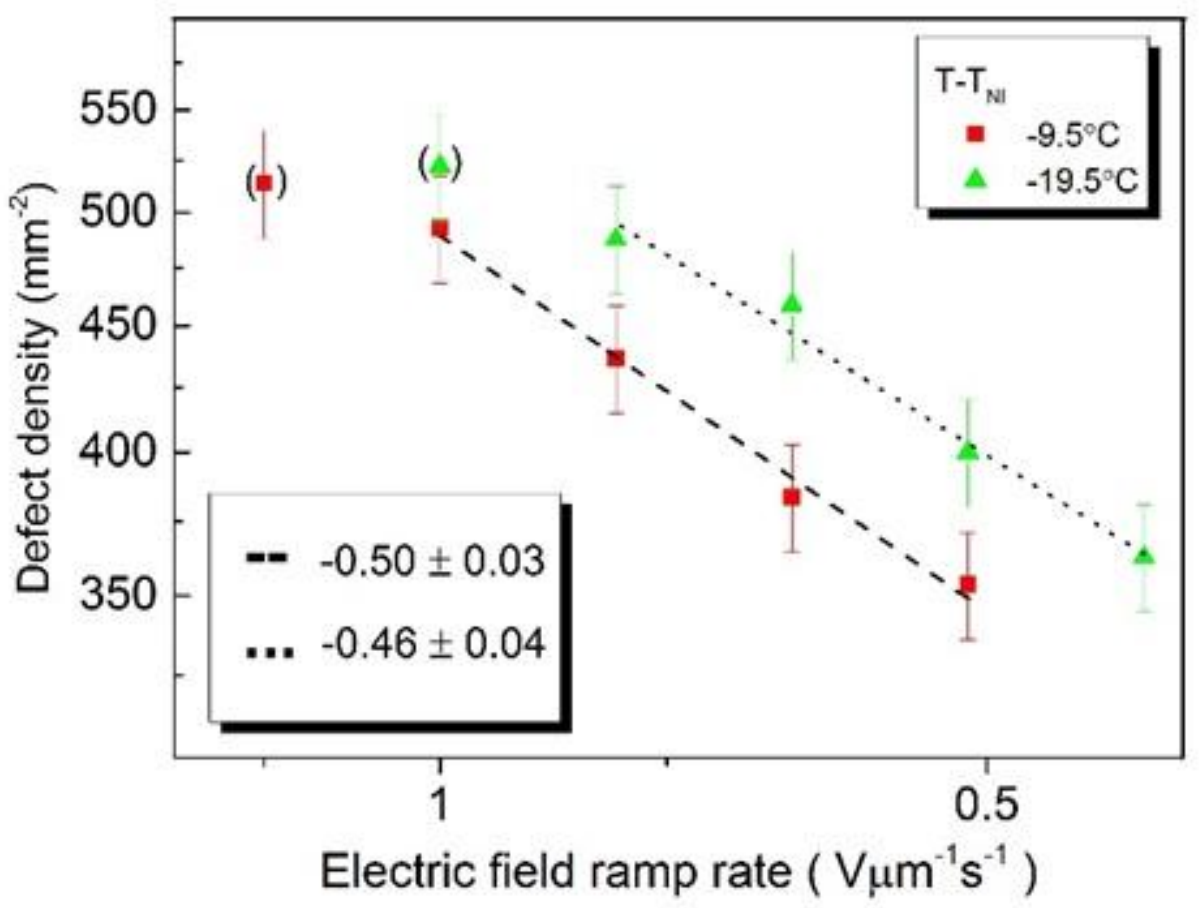


Figure 5. Defect annihaltion at higher electric field ramp rates can be partially alleviated by repeating the experiment with a shorter delay time $(t=0.1 \mathrm{~s})$ allowing for the exponent for defect formation to be calculated.

A different liquid crystal, N-(4-Methoxybenzylidene)-4-butylaniline (MBBA), was utlilised to verify the repeatability of the scaling law of eq.(1) and to demonstrate independence of the system investigated. MBBA has a negative dielectric anisotropy of $\Delta \varepsilon=-0.56$ and the following phase sequence on cooling: Isotropic $43{ }^{\circ} \mathrm{C}$ Nematic $17{ }^{\circ} \mathrm{C}$ Crystal. The results of experiments performed accoring to the procedure outlined above are depicted in figure 6 . The measured scaling exponent of $\alpha=-1 / 2$ is in agreement with the predicted value and the results of figure 4 . The range of ramp rates over which scaling is observed is smaller than for ZLI-2806. This is due to a slower defect formation process and thus a shortened time between the formation of easily identifiable defects and their annihlation. Also here, the scaling exponent for defect formation is independent of temperature, $\alpha \neq \alpha(T)$, as depicted in the inset of figure 6 .

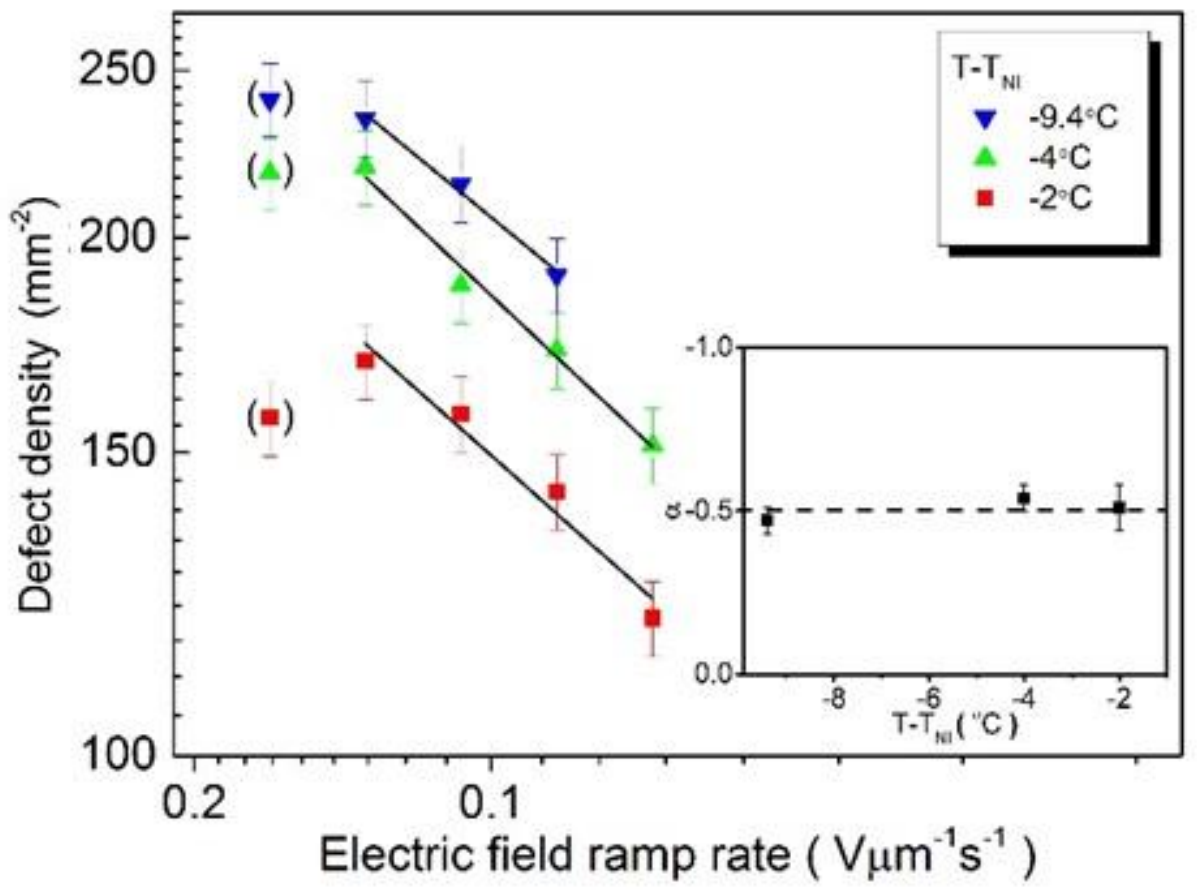


Figure 6. Defect density after defect formation in MBBA at three different temperatures as a function of the applied electric field ramp rate. The scaling exponent $\alpha$ is independent of reduced temperature $T-T_{N 1}$ as demonstrated in the inset of the figure.

The formation of umbilical defects induced by a ramped electric field in a homeotropically aligned nematic liquid crystal with a negative dielectric anisotropy was investigated. The system undergoes a continuous texture transition at constant temperature within the nematic state, accompanied by a change from a uniform state to a defect dominated state. At longer times, the formed defects annihilate in a process which has extensively been studied before. Here, on the other hand, we experimentally demonstrate for a liquid crystalline system that also the defect formation follows predicted scaling laws. The density of defects formed was shown to scale with the rate of change of the control parameter, in our case the electric field ramp rate, according to the scaling law $\rho \propto \tau_{Q}{ }^{\alpha}$, with an exponent $\alpha=-1 / 2$, in agreement with the prediction made by Zurek ${ }^{4}$. Any deviation from the predicted scaling behaviour was shown to be the result of defect annihilation which would begin at some time after defect formation. The scaling law was validated by comparing defect formation over a range of temperatures as well as using two different liquid crystal materials.

Valuable discussions with T. Galla and T. Mullin are gratefully acknowledged.

[1] N.D Mermin, Rev. Mod. Phys. 1979, 51, 591.

[2] T.W.B Kibble, J. Phys. A 1976, 9, 1387.

[3] T.W.B. Kibble, Phys. Rep. 1980, 67, 183.

[4] W.H. Zurek, Nature 1985, 317, 585.

[5] C. Liu, M. Muthukumar, J. Chem. Phys. 1997, 106, 7822.

[6] N.D. Antunes, L.M.A. Bettencourt, W.H. Zurek, Phys. Rev. Lett. 1999, 82, 2824.

[7] A. Yates, W.H. Zurek, Phys. Rev. Lett. 1998, 80, 5477.

[8] I. Chuang, B. Yurke, R. Durrer, N. Turok, Science 1991, 251, 1336. 
[9] I. Dierking, O. Marshall, J. Wright, N. Bulleid, Phys. Rev. E 2005, 71, 061709.

[10] C. Bäuerle, Y.M. Bunkov, S.N. Fisher, H. Godfrin, G.R. Pickett, Nature 1996, 382, 332.

[11] V.M. Ruutu, V.B. Eltsov, M. Krusius, Y.G. Makhlin, B. Placais, G.E. Volovik Phys. Rev. Lett. 1998, 80, 1465.

[12] P. Hendry, N. Lawson, R. Lee, P. McClintock, C. Williams, Nature 1994, 368, 315.

[13] M.E. Dodd, P.C. Hendry, N.S. Lawson, P.V.E McClintock, C.D.H Williams, Phys. Rev. Lett. $1998,81,3703$.

[14] E. Kavoussanaki, R. Monaco, R.J. Rivers, Phys. Rev. Lett. 2000, 85, 3452.

[15] S. Ulm, J. Rossnagel, G. Jacob, C. Degunther, S.T. Dawkins, U.G. Poschinger, R. Nigmatullin, A. Retzker, M.B. Plenio, F. Schmidt-Kaler, K. Singer, Nature Communications 2013, 4, 2290.

[16] S. Ducci, P.L. Ramazza, W. Gonzales-Vinas, F.T. Arecchi, Phys. Rev. Lett. 1999, 83, 5210.

[17] R. Monaco, J. Mygind, M. Aaroe, R.J. Rivers, V.P. Koshelets, Phys. Rev. Lett. 2006, 96, 180604.

[18] S.C. Chae, N. Lee, Y. Horibe, M. Tanimura, S. Mori, B. Gao, S. Carr, S.-W. Cheong, Phys. Rev. Lett. 2012, 108, 167603.

[19] A. Bogi, P. Martinot-Lagarde, I. Dozov, M. Nobili, Phys. Rev. Lett. 2002, 89, 225501.

[20] P. Oswald, J. Ignes-Mullol, Phys. Rev. Lett. 2005, 95, 027801.

[21] I. Dierking, M. Ravnik, E. Lark, J. Healey, G.P. Alexander, J.M. Yeomans, Phys. Rev. E, 2012, 85, 021703.

[22] L. Radzihovsky, Phys. Rev. Lett. 2015, 115, 247801.

[23] R. Stannarius, K. Harth, Phys. Rev. Lett. 2016, 117, 157801.

[24] A. del Campo, W.H. Zurek, Int. J. Mod. Phys. A 2014, 29, 1430018 .

[25] P.G. De Gennes, J. Prost, The Physics of Liquid Crystals 2nd Ed. (Oxford University Press, Oxford 2008).

[26] A. Rapini, J. Phys. Paris 1973, 34, 629.

[27] A. Saupe, Mol. Cryst. Liq. Cryst. 1973, 21, 211.

[28] R.B. Meyer, Mol. Cryst. Liq. Cryst. 1972, 16, 355. 